\title{
Reflets
}

Revue d'intervention sociale et communautaire

\section{D’intervenante à militante féministe : défi et stratégies à la Maison Unies-Vers-Femmes}

\section{Sophiane Demers-Tessier}

Volume 23, numéro 1, printemps 2017

URI : https://id.erudit.org/iderudit/1040755ar

DOI : https://doi.org/10.7202/1040755ar

Aller au sommaire du numéro

Éditeur(s)

Reflets, Revue d'intervention sociale et communautaire

ISSN

1203-4576 (imprimé)

1712-8498 (numérique)

Découvrir la revue

Citer cet article

Demers-Tessier, S. (2017). D’intervenante à militante féministe : défi et stratégies à la Maison Unies-Vers-Femmes. Reflets, 23(1), 212-218.

https://doi.org/10.7202/1040755ar
Résumé de l'article

Oeuvrant en maison d'hébergement auprès de femmes et d'enfants victimes de violence conjugale, dans une perspective féministe, les intervenantes de la Maison Unies-Vers-Femmes (MUVF) doivent occuper le rôle d'agente de changement social, tout en effectuant des interventions d'urgence et de proximité. Cette double responsabilité pose un défi de taille qui nécessite diverses stratégies de gestion et d'intervention qui seront abordées dans cet article.
Tous droits réservés (C) Reflets, Revue d'intervention sociale et communautaire, 2017
Cecument est protégé par la loi sur le droit d'auteur. L'utilisation des services d'Érudit (y compris la reproduction) est assujettie à sa politique d'utilisation que vous pouvez consulter en ligne. 


\title{
D'intervenante à militante féministe : défi et stratégies à la Maison Unies-Vers-Femmes
}

\author{
Sophiane Demers-Tessier \\ Intervenante responsable du volet défense de droits individuels et collectifs \\ Maison Unies-Vers-Femmes
}

\section{Résumé}

Cuvrant en maison d'hébergement auprès de femmes et d'enfants victimes de violence conjugale, dans une perspective féministe, les intervenantes de la Maison Unies-VersFemmes (MUVF) doivent occuper le rôle d'agente de changement social, tout en effectuant des interventions d'urgence et de proximité. Cette double responsabilité pose un défi de taille qui nécessite diverses stratégies de gestion et d'intervention qui seront abordées dans cet article.

Mots clés : violence conjugale, féminisme, militantisme, urgence, première ligne, maison d'hébergement

\section{Abstract}

Working in a domestic violence shelter, with a feminist approach, means that employees have to work toward social change while dealing with crisis situations. This dual role is sometimes difficult and requires multiple strategies which will be discussed in the present article.

Key words: domestic violence, feminism, activism, emergency, front line, women shelter

\section{Introduction}

Les maisons d'hébergement du Québec sont des organismes de première ligne, offrant un hébergement sécuritaire et soutenant aux femmes victimes de violence conjugale et à leurs enfants. Dans la plupart des maisons, les services sont disponibles à tous moments 
de la journée, et ce, toute l'année. Les intervenantes travaillent auprès des femmes et des enfants dans leur vie quotidienne, le jour, le soir, la nuit, tant en semaine que durant la fin de semaine.

Certaines maisons œuvrent dans une perspective féministe, c'est-à-dire qu'elles visent la reprise de pouvoir des femmes et des enfants ainsi qu'un changement structurel favorisant l'égalité homme-femme. Leur but ultime est de contrer la violence conjugale individuellement, collectivement et socialement.

La Maison Unies-Vers-Femmes (MUVF) de Gatineau est une maison d'hébergement qui prône l'approche féministe. Les intervenantes qui y œuvrent occupent alors les rôles d'intervenante en milieu de vie et de militante. Le présent article a pour objectif de démontrer le défi que pose l'association de ces deux rôles, ainsi que les stratégies mises en place par l'équipe pour le relever.

\section{Mise en contexte}

Fondée en 1979, la MUVF est une maison d'aide et d'hébergement pour les femmes victimes de violence conjugale et leurs enfants. Ayant pour mission de contrer la violence conjugale, elle œuvre auprès des femmes et des enfants afin de faciliter leur reprise de pouvoir sur leurs vies à la suite des agressions subies. Pour ce faire, les intervenantes offrent un accueil inconditionnel, du soutien individuel face-à-face et par téléphone, ainsi que des groupes de soutien et d'éducation.

Dans les dernières années, la MUVF a créé quatre postes afin de gérer les situations, réflexions et projets divers (vie communautaire et cheminement des femmes; jeunesse; défense de droits individuels et collectifs; éducation populaire et sensibilisation). Depuis peu, la MUVF a aussi une coordonnatrice à l'intervention, qui guide l'équipe composée de 15 intervenantes sur le terrain. Puis, pour assurer son fonctionnement, la MUVF a une directrice générale, qui supervise l'ensemble des activités, ainsi qu'une responsable administrative et un conseil d'administration.

Toutes les intervenantes travaillent dans l'urgence, c'est-à-dire qu'à tout moment, elles peuvent recevoir l'appel ou la visite de femmes et d'enfants ayant besoin de sécurité. Tous les jours, de nouveaux défis se posent selon les manques, les besoins, les réussites et le cheminement de chaque famille fréquentant la maison. Les intervenantes sur le terrain doivent alors se concentrer afin de vivre le moment présent avec les femmes et les enfants, tout en les soutenant dans leurs besoins à court, à moyen et à long terme. 


\section{Défi}

La mission de contrer la violence conjugale se fait d'abord et avant tout dans le processus de reprise de pouvoir individuel et collectif des femmes et des enfants ayant été victimes de violence conjugale. Toutefois, œuvrant dans une perspective féministe, les intervenantes de la MUVF situent cette mission dans un cadre beaucoup plus large, s'appuyant sur la définition de l'Organisation des Nations Unies (ONU) formulée en 1993 (reprise par le gouvernement du Québec dans sa politique de 1995), qui campe cette problématique dans la domination masculine :

Le problème de la violence conjugale s'inscrit dans la problématique plus large de la violence faite aux femmes. Dans sa déclaration sur l'élimination de la violence contre les femmes, adoptée le $1^{\text {er }}$ décembre 1993, l'Assemblée générale des Nations Unies a reconnu que cette violence est la manifestation de rapports de force historiquement inégaux qui ont abouti à la domination des hommes sur les femmes. (Gouvernement du Québec, 1995)

Cela signifie que la violence conjugale est une problématique sociale, ancrée dans le patriarcat et maintenue par le capitalisme, qui encore aujourd'hui fait des ravages dans la vie des femmes et des enfants. Pour contrer cette injustice sociale, il faut alors beaucoup plus qu'offrir des interventions directes auprès des femmes. Il faut s'allier, dénoncer les injustices et revendiquer un changement de mentalité et un respect des droits et des ressources répondant aux besoins des femmes et des enfants. C'est pourquoi les intervenantes de la MUVF se perçoivent comme des agentes de changement social, à tous les jours et dans toutes leurs interventions.

Or, un défi important se pose pour les travailleuses de la MUVF : offrir des services de première ligne, tout en militant pour la cause des femmes et des enfants. En effet, il est difficile de prendre du temps pour réfléchir et analyser des problématiques, se poser et agir pour les enrayer, tout en intervenant dans un milieu de vie où les femmes et les enfants ont des besoins immédiats en lien avec leur bien-être et leur sécurité.

Afin de maintenir le militantisme au cour de la mission de la MUVF, les intervenantes et la direction ont d'abord priorisé les enjeux sociaux sur lesquels elles se penchent, ainsi que les actions à mener, puis elles ont modifié les attentes envers les intervenantes, se sont alliées à d'autres organismes et ont jumelé le militantisme à l'intervention quotidienne. 


\section{Priorisation des enjeux et des actions}

En 2014, la MUVF a fait un « retour aux valeurs féministes » (Paradis et Côté, 2015), ce qui lui a permis de remettre la situation des femmes et des enfants au cœur des actions posées par l'organisme. De cette façon, la défense des droits prend de plus en plus d'importance dans les interventions.

En ce sens, soutenues par la direction, la coordonnatrice et l'intervenante responsable du volet défense de droits individuels et collectifs, les travailleuses ont décidé de se pencher sur deux enjeux importants sur lesquels s'appuient leurs revendications : la violence faite aux femmes et la pauvreté des femmes. Afin d'agir sur ces problèmes sociaux, la MUVF a déterminé des moments de l'année cruciaux pour prendre place dans l'espace public, tels que les 12 jours d'actions contre la violence faite aux femmes, la journée internationale des droits des femmes, la semaine québécoise contre l'intimidation et la violence à l'école, et la Journée d'action contre la violence sexuelle faite aux femmes. De cette façon, les travailleuses peuvent se préparer à l'avance et jouer leur rôle d'agentes de changement social, malgré l'intervention d'urgence qui pèse lourd dans leurs tâches quotidiennes. Par exemple, chaque année, elles planifient une vigile commémorant les victimes de la tuerie de Polytechnique, ainsi que l'ensemble des femmes assassinées par des hommes. Il s'agit d'un rendez-vous annuel pour sensibiliser, dénoncer et revendiquer un changement social.

En ce qui a trait à la pauvreté, la MUVF accorde une grande importance à la lutte contre l'austérité. Constatant qu'il faut s'opposer à l'austérité puisqu'elle fait des ravages dans la vie des femmes et des enfants, la direction libère des intervenantes de leurs tâches quotidiennes afin qu'elles se rendent aux différentes manifestations régionales et provinciales, tout en s'assurant qu'au moins une d'entre elles est toujours présente pour les femmes et les enfants.

De manière plus large et pour lutter simultanément contre la violence faite aux femmes et la pauvreté, la MUVF a décidé, en 2016, d'orienter entièrement son plan d'action bisannuel vers la défense de droits des femmes et des enfants. Ainsi, toutes les intervenantes doivent développer des projets et se mobiliser pour faire pression sur la société patriarcale. Il s'agit alors d'une stratégie pour permettre à l'équipe de se réserver du temps pour planifier et participer aux activités militantes que nécessitent ces luttes. 


\section{Modification des attentes envers les employées}

Avec la restructuration de la MUVF, en 2014, les attentes envers les employées ont grandement changé. D’abord, l'aspect militant des personnalités des intervenantes doit ressortir dans les entrevues pour qu'elles soient embauchées. Certaines compétences sont ainsi essentielles :

Encourager et canaliser le sentiment d'outrage; collectiviser le vécu et les injustices; développer un intérêt et une capacité d'analyse sociale pour comprendre les enjeux du monde avec un parti pris pour l'égalité hf et la justice sociale; s'indigner face aux injustices; sortir de sa propre victimisation et impuissance face au changement social; déconstruire les préjugés, les nôtres, ceux des collègues, ceux des femmes VVC et ceux $\mathrm{p} / \mathrm{r}$ aux femmes VVC. (RMFVVC, 2016)

En exigeant ces attitudes et habiletés dès l'embauche, la MUVF s'assure que les nouvelles intervenantes seront au même diapason que l'équipe de travail. Elles pourront alors prendre part aux réflexions et au militantisme de l'équipe, ainsi qu'à une démarche collective de dévictimisation, afin de contrer leur propre impuissance en tant que femmes.

Par ailleurs, pour miser encore plus sur l'importance du militantisme, la direction a décidé de modifier le titre que portent les employées. Plutôt que travailleuses ou intervenantes, elles sont dorénavant nommées intervenantes militantes féministes (IMF) dans tous les documents légaux et le langage quotidien de la MUVF. Cela signifie qu' elles doivent d'emblée s'afficher comme étant féministes et démontrer que le changement social est aussi important que le changement individuel dans leur travail auprès des femmes victimes de violence conjugale.

\section{Alliance : coalition et concertation}

Qui dit féminisme dit alliance et solidarité. Depuis plusieurs années, les femmes ont fait avancer leur situation en créant un mouvement militant fort, empreint de revendications communes. Les IMF de la MUVF se rallient à ce principe selon lequel l'union fait la force. Pour mener leurs luttes, elles forment alors des concertations et des coalitions avec différents acteurs et actrices du milieu de la santé et des services sociaux ainsi que des citoyennes et citoyens militants. De cette façon, la MUVF peut prendre le temps de 
formuler ses revendications et les exposer sur la place publique, tout en offrant un service continu aux enfants et aux femmes victimes de violence conjugale.

Deux exemples de pratiques peuvent illustrer ces alliances. D'abord, la directrice de la MUVF siège à une table de concertation avec des policiers pour clarifier des situations problématiques et veiller à ce qu'ils offrent un service approprié assurant la sécurité des femmes victimes de violence conjugale. Il s'agit alors d'un moyen direct de faire valoir les droits des femmes et de dénoncer les injustices qu'elles subissent. Puis, en ce qui a trait à la coalition, l'un des exemples est la participation d'une IMF au comité organisateur de la Marche mondiale des femmes. Il s'agit d'un groupe de femmes formant une "alliance politique " face à des «adversaires communs » dans le but de " porter des revendications » (RMFVVC, 2016).

\section{Le militantisme au quotidien}

Afin d'assurer une réelle cohérence entre le militantisme féministe et l'intervention en milieu de vie, les IMF mettent en œuvre diverses stratégies visant à alimenter l'esprit critique des femmes et leur désir de changement collectif, entre autres par le biais de la conscientisation.

En maison, par exemple, la vie communautaire, l'éducation populaire autonome, l'intervention de groupe et les accompagnements sont les points de départ d'une défense de droits individuelle et collective (RMFVVC, 2016). Ceci dit, la vie associative des femmes et des enfants est considérée comme un lieu privilégié de collectivisation et d'analyse sociale. En intervention de groupe, formelle ou informelle, les IMF amènent des sujets d'actualité afin de susciter la discussion. Ces discussions mènent à l'éducation populaire autonome, c'est-à-dire :

L'ensemble des démarches d'apprentissage et de réflexion critique par lesquelles des citoyens et citoyennes mènent ensemble des actions qui provoquent une prise de conscience individuelle et collective au sujet des conditions de vie ou de travail, et qui visent à court ou à long terme une transformation sociale, économique, culturelle et politique de leur milieu. (MÉPACQ, 2016)

Dans la prochaine année, l'intervenante responsable du volet défense de droits individuels et collectifs mettra sur pied un groupe de militantes ayant déjà reçu des services 
de la MUVF. Sorties de relations de violence conjugale, ces femmes pourront se mobiliser et offrir une voix à celles qui sont encore aux prises avec des situations dangereuses et préjudiciables et qui souhaitent s'en sortir.

\section{Conclusion}

En somme, le militantisme féministe en maison d'hébergement pose des défis de taille. Il n'est pas facile pour les IMF de jongler entre l'intervention d'urgence et la défense de droits collectifs. Toutefois, ayant conscience de la portée sociale de leur travail, elles font tout en leur pouvoir pour favoriser un monde plus juste et égalitaire pour les femmes. Pour les trois prochaines années, la MUVF continuera à développer des stratégies pour favoriser la reprise de pouvoir collective des femmes et des enfants. Elle mettra de l'avant le militantisme féministe, et ce, "tant qu'il le faudra ".

\section{Bibliographie}

CÔTÉ, Isabelle, et Véronique PARADIS (2015). «Un retour aux valeurs féministes en maison d'hébergement pour femmes victimes de violence conjugale : la réflexion de l'équipe de la Maison Unies-Vers-Femmes de Gatineau ", Reflets: revue d'intervention sociale et communautaire, Vol. 21, No 1, p. 197-205.

GOUVERNEMENT DU QUÉBEC (1995). Politique d'intervention en matière de violence conjugale, réf. du 7 septembre 2016,

http://www.scf.gouv.qc.ca/fileadmin/publications/Violence/Prevenir_depister_contrer_Politique_ VC.pdf

MOUVEMENT D'ÉDUCATION POPULAIRE ET D'ACTION COMMUNAUTAIRE DU QUÉBEC (2016). Quelques définitions, réf. du 31 août 2016,

http://www.mepacq.qc.ca/education-populaire-et-luttes-sociales/quelques-definitions/

REGROUPEMENT DES MAISONS POUR FEMMES VICTIMES DE VIOLENCE CONJUGALE (2016). L'éducation populaire autonome, [Document de formation : La défense collective des droits, c'est l'affaire de toutes!], Montréal, 12 p.

REGROUPEMENT DES MAISONS POUR FEMMES VICTIMES DE VIOLENCE CONJUGALE (2016). Les postures d'intervention, [Document de formation : La défense collective des droits, c'est l'affaire de toutes!], Montréal, 4 p. 